\title{
Investigation of co-travelling solitary wave collisions in a granular chain
}

\author{
Paul Anzel* and Chiara Daraio \\ Department of Applied Physics and Material Science, California Institute of Technology, \\ 1200 E California Ave, MC 105-50, Pasadena, CA, 91125
}

\begin{abstract}
We present investigations into the collision of co-travelling solitary waves in a granular chain. Impulses are injected into the system by means of a piezo stack and the results are compared to a numerical model of discrete masses connected by non-linear springs. Similar to other solitary wave-carrying systems, a phase shift in both interacting solitary waves is observed due to their collision. Additionally, the formation of small secondary waves is observed in both numerical and experimental results. Insight into solitary wave interactions will be important for high-frequency excitation of a granular crystal, which may allow for improved Non-Destructive Evaluation (NDE) and Structural Health Monitoring (SHM) methods.
\end{abstract}

\section{KEYWORD LIST}

Solitary wave, Hertzian chain, granular media, phase shift

\section{INTRODUCTION}

The dynamics of a one-dimensional array of elastic spheres has proven to be a rich source of interesting physical phenomena. Instead of the traditional dispersive wave motion seen in most media, the dominant form of energy and momentum transmission in this system is instead in the form of solitary waves. For a system of particles just barely in contact, the velocities of the particles are described by the equation:

$v=\left(\frac{25}{16}\right)\left(\frac{V_{\text {sol }}^{5}}{c^{4}}\right) \cos ^{4}\left(\frac{\sqrt{10}}{10 R}\left(x-V_{\text {sol }} t\right)\right)$

with position $x$, time $t$, wave velocity $V_{\text {sol, }}$, sphere radius $R$, and coefficient $c^{2}=2 E / \pi \rho\left(1-v^{2}\right)$ based on material parameters of the spheres $(E, \rho$, and $v$ are Young's modulus, density, and Poisson ratio respectively). Only one hump of the cosine solution is kept and the velocity is 0 otherwise, yielding a localized, collective motion of particles about five spheres long ${ }^{1-3}$.

The presence of solitary waves in this system stems from the non-linear (Hertzian) contact force between two elastic spheres ${ }^{4}$. When two spheres are pressed statically into each other, the restitutional force grows as the overlap to the $3 / 2$ 's power, even for infinitesimally small displacements. The equation of motion for particle $n$ is given by:

$m \ddot{x}_{n}=A_{c}\left[\delta_{n}\right]_{+}^{3 / 2}-A_{c}\left[\delta_{n+1}\right]_{+}^{3 / 2}$

Here $R$ is the radius of the sphere, $\delta_{n}=2 R-\left(x_{n-1}-x_{n}\right)$ is the overlap between two spheres, and [y $]_{+} \operatorname{means} \max (\mathrm{y}, 0)$ (there is no tensional force between two spheres). The constant of proportionality $A_{c}$ is thus:

${ }^{*}$ E-mail address: panzel@caltech.edu

Smart Sensor Phenomena, Technology, Networks, and Systems Integration 2012,

edited by Theodore E. Matikas, Kara J. Peters, Wolfgang Ecke, Proc. of SPIE

Vol. 8346, 83460G · (C) 2012 SPIE · CCC code: 0277-786X/12/\$18 · doi: 10.1117/12.917474

Proc. of SPIE Vol. 8346 83460G-1 
$A_{c}=\frac{E \sqrt{2 R}}{3\left(1-v^{2}\right)}$

For a single solitary wave, the maximum force $\mathrm{F}_{\mathrm{m}}$ between two particles is related to the maximum particle velocity $V_{m}=\left(25 V_{\text {sol }}^{5} / 16 c^{4}\right)$ by $^{5}:$

$V_{m}=\left(\frac{6}{5 \pi \rho}\right)^{1 / 2}\left(\frac{3\left(1-v^{2}\right)}{4 E R^{5}}\right)^{1 / 3} F_{m}^{5 / 6}$

The presence of dissipative losses is ignored in our numerical calculations, but is a factor for our experimental work, slowing down solitary waves as they progress. A number of different models for damping have been proposed ${ }^{6-8}$, but none provide a full physical description of the damping observed.

As is evident from equation 1, the propagation speed $V_{\text {sol }}$ of a wave is dependent upon its amplitude with larger pulses moving faster, and a wave located behind a smaller one will eventually overtake and interact with it. The investigation of solitary wave collisions in a granular chain has been a subject of active research in the past decade. Numerical simulations by Manciu, et al. ${ }^{9}$ have noted that the head-on collision of two equal waves results in the formation of secondary solitary waves (SSWs) due to the lack of tensional forces and the discreteness of the system: the spheres break away from each other and as they regain contact their collisions generate new waves. Santibanez, et al. ${ }^{10}$ have experimentally confirmed the creation of these waves in an equal head-on collision, as well as noting that the waves undergo a phase advance that can be described as the interaction of two quasiparticles. Simulations by Zhen-Ying, et al. ${ }^{11}$ have shown that head-on and co-travelling collisions of solitary waves of unequal magnitude result in the exchange of energy between the solitary waves as well as the formation of secondary waves.

While Nesterenko has noted ${ }^{3}$ that the co-travelling interaction of two solitary waves induces a phase shift in both signals, no systematic study of the phase advances and delays experienced by solitary waves during a co-travelling collision has been undertaken. In this paper, we present numerical investigations into these phase changes and we perform experimental measurements of this phenomenon. In addition, we provide experimental confirmation of the formation of secondary solitary waves.

\section{EXPERIMENTAL SETUP}

The experimental apparatus consists of a 1-dimensional chain of $5119.05 \mathrm{~mm}$ diameter hardened 440C stainless steel spheres (McMaster-Carr). These spheres have density $\rho=7650 \mathrm{~kg} / \mathrm{m}^{3}$, Poisson's ratio $v=0.27$, and Young's modulus $E$ $=200 \mathrm{GPa}^{12}$. The spheres rest between two polycarbonate rods covered with Teflon tape to reduce friction and are held at an incline of 2 degrees with a weak spring at the end to ensure that all spheres are in held in contact. Instrumented sensors (particles with a thin layer of PZT ceramic inside, more details on their construction and use can be found in reference 13) are placed at the 7th and 45th position in the chain. The mass of these instrumented particles is nearly that of the regular particles, so propagation in the chain is largely unaffected by their presence. A schematic diagram of the experimental setup is provided in figure 1(a).

To provide reference signals for determining phase changes the piezo was given a single voltage pulse at a number of different levels to generate single waves whose amplitude and time of flight between the two sensors were measured. Next, to investigate the collision of the two waves, the two pulsers are used in conjunction. One pulser is first activated, driving the piezo to $150 \mathrm{~V}$ in order to create the smaller signal, and the other is then subsequently triggered at some later time to drive the piezo to a higher voltage (between 330-420 V), generating the larger pulse. The timing between the activation of the two pulser units was set so the interaction between the two waves occurs between the two instrumented particles, and only runs where the two pulses were distinctly separated at each sensor were kept. The phase changes were determined by finding the time of flight of the waves between the two sensors and subtracting the time of the reference 
signal with the closest amplitude. Both the amplitude and time position of each wave is determined by taking a quadratic fit to the 100 points near its peak (a regime where the waves are well described with a parabola).

a)

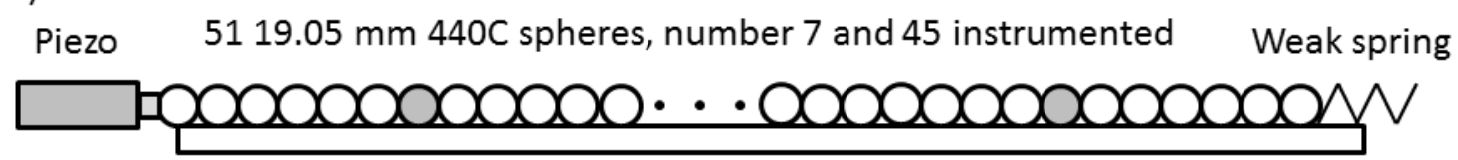

2 polycarbonate rods with Teflon coating

b)

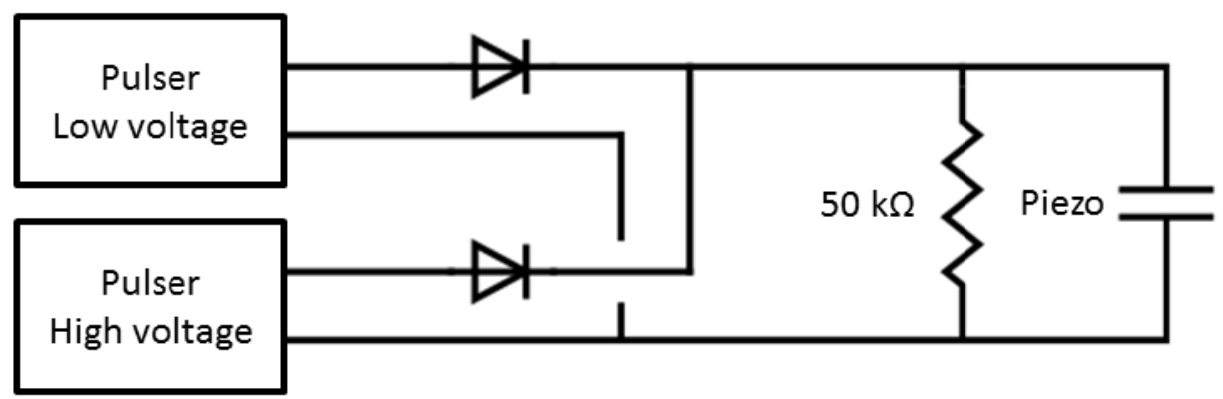

Figure 1: (a) Schematic diagram of the experimental set-up. The pulses are excited on the left of the chain by the piezo actuator. The propagating waves are detected by instrumented particles (shaded gray circles). (b) Driving circuit configuration.

To actuate the system, a piezo-stack (Piezomechanik PIA 1000/10/25 VS20) is held at one end and is rapidly ramped up to high voltages to provide impulses. The voltage jumps are provided with two pulser units (Piezomechanik HVPs) which drive the piezo to chosen high voltages (voltage rise time $<1 \mu \mathrm{s}$ ). Diodes are placed in front of each pulser unit to ensure all current goes to the piezo and keep it at the desired high voltages and the piezo is bridged with a resistor to slowly bleed the voltage off (decay time $4.3 \mathrm{~ms}$ ) to zero out the system for subsequent runs. A schematic of the driving circuit configuration is provided in figure $1(\mathrm{~b})$.

To estimate the experimental error in the phase advances and delays, the time of flight versus the amplitude of the reference signals were fit with a power-law (which gave a good fit for the time of flights) and the standard deviation of the residuals was calculated, yielding an error of about $3.8 \mu$ s for the reference signal time of flight. A similar error is predicted for the times of flight of the colliding solitary waves, yielding a total estimated error of $5.4 \mu$ s for each measurement.

Amplitude measurements of secondary solitary waves at the second sensor were taken when these waves were observed to have clearly separated from the two primary waves. Our ability to resolve these waves was primarily limited by the resolution of our DAQ board, which gives a $0.064 \mathrm{~N}$ force resolution.

\section{NUMERICAL MODEL}

We modeled the interaction of the two co-travelling solitary waves using a discrete particle model based on solving equation $(2)^{14}$. Spheres are treated as point masses connected by non-linear springs with material parameters given in the experimental section. The equations of motion of the spheres are solved using a $4^{\text {th }}$ order Runge-Kutta solver ${ }^{15}$. For most simulations, the time step was set to be $1 \mu \mathrm{s}$, which conserves energy to about one part in five hundred thousand in our simulations and gives excellent agreement in phase change values with simulations using smaller time steps. When the 
large wave was at least twelve times larger than the smaller one (giving computed time changes on the order of several microseconds or less) the time step was reduced to $0.1 \mu \mathrm{s}$.

Looking at the velocities of each sphere starting from the beginning of the chain, we define the point where the solitary waves are beginning to collide as when the minimum velocity experienced by the sphere between the two waves passes a threshold of $1 \%$ of its maximum velocity when the small wave is centered on it. A similar criterion is used to determine when the two waves have separated. The location and size of each wave was determined using a quadratic fit around its maximum. The expected position of a solitary wave - from which the phase change can be determined - is found by taking a linear fit of the positions of the wave for the five spheres before the two begin two collide, and then subtracting the time of the solitary wave post-collision from the expected time from the fit. The waves are actuated by a driven simple harmonic oscillator, which models the motion of the piezo-stack used to drive the experimental set-up ${ }^{16}$.

To determine the expected secondary solitary wave forces, the bead forces at the threshold of separation described above were extracted. While the force amplitudes of the secondary waves grow slightly as they move away from their point of generation, this was thought to give the best estimate for the forces observed in experiment, where the solitary waves are generally just separating from each other once they reach the second sensor.

Previous numerical studies ${ }^{11}$ have shown that while the energy exchanged between two solitary waves does show some dependence upon the point of interaction (whether they start colliding in the middle of a sphere, at the point of contact between two spheres, or somewhere in between) the effect is on the order of a fraction of a percent. Simulations run with different time delays between pulses have shown that this does not change the amount of phase advance or delay to any appreciable amount.

\section{RESULTS}

\subsection{Numerical results}

As can be expected from soliton-soliton interactions in other systems ${ }^{17}$, the collision of the two solitary waves leads to a time delay for the smaller wave and a time advance for the larger one. For our work, the maximum velocities $V_{i}$ (with subscripts $l$ and $s$ corresponding to the large and small waves, respectively) of the waves are used to define the wave's amplitudes. Nesterenko has remarked ${ }^{1}$ that non-dimensionalizing the equations of motion for an uncompressed chain with an impacting sphere of initial velocity $V_{0}$ gives a unit-less time $\tau=t\left(A_{c} / m\right)^{2 / 5} V_{0}^{1 / 5}$. The amplitude of a wave is proportional to the impacting velocity ${ }^{18}$, so we expect the time advance and delay $(\Delta t)$ of the small and large solitary waves to scale as $V_{l}^{-1 / 5}$ and look for solutions of the form:

$\Delta t_{i}=V_{l}^{-1 / 5} F_{i}\left(V_{l} / V_{S}\right)(5)$

for some unknown functions $F_{l}$ and $F_{s}$. The numerical results for both the large and small waves are presented in figure 2, with the large wave ranging in size from one percent larger than the smaller one to one hundred times its size. Note that the data for the smaller wave is truncated at $V_{l} / V_{s}=22.75$, as above this point the results for the smaller wave were began to spread from a single line and were deemed unreliable, likely because the smaller wave's loss of energy also starts coming into play. 

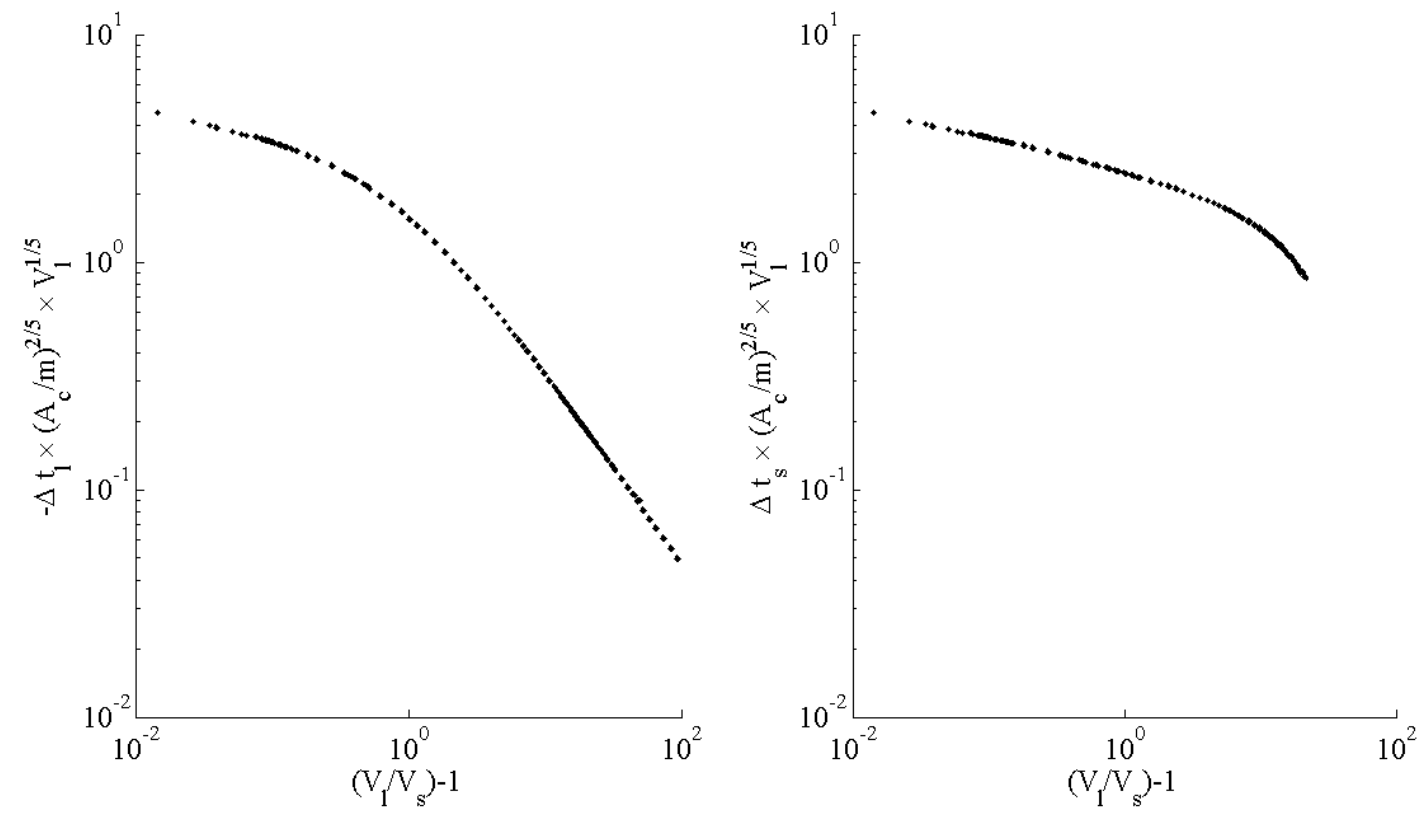

Figure 2: Numerically determined time changes of large and small solitary waves (a and b, respectively). Note that four data sets with different values of $\mathrm{V}_{\mathrm{s}}$ are plotted in both figures.
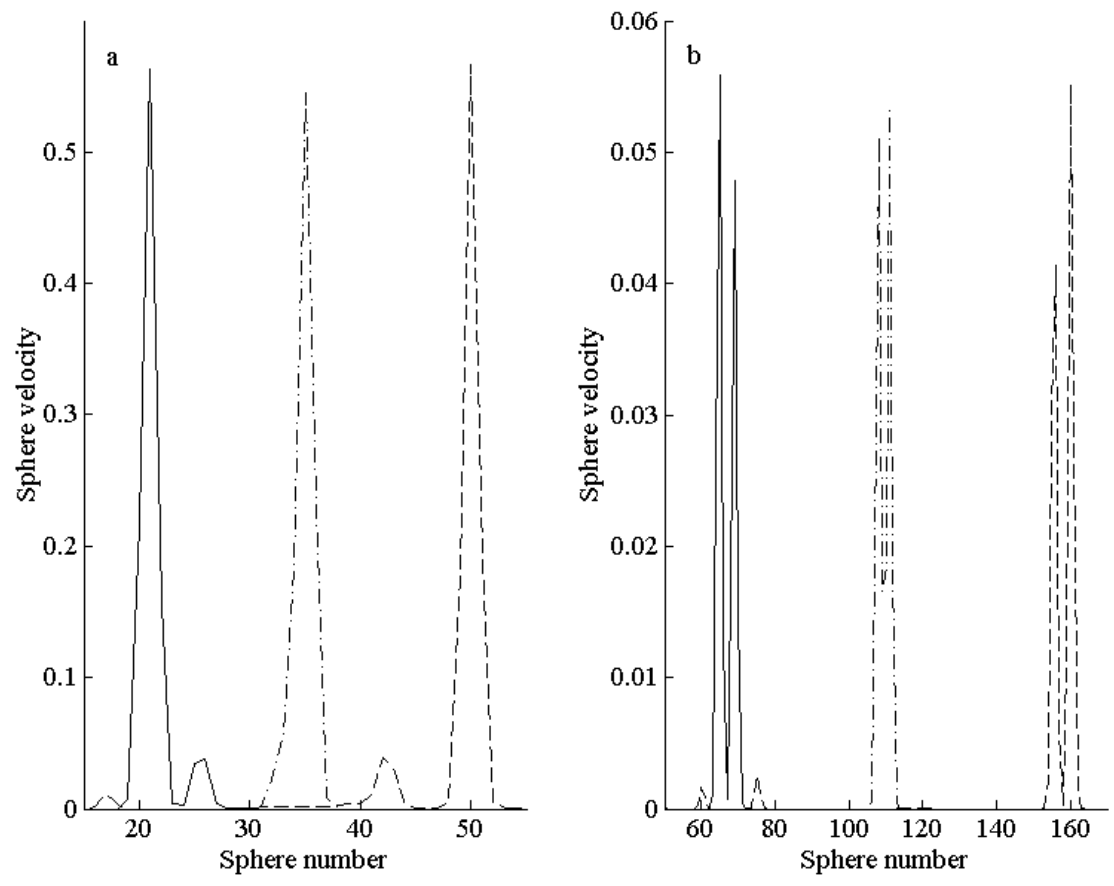

Figure 3: Snapshots of sphere velocities in the two limiting regimes of solitary wave interaction. If the larger solitary wave is much bigger than the smaller one (a) the two overlap and we can treat the secondary wave as an area of localized compression. If the two are of comparable size (b) the two waves remain distinct and the interaction can be treated as the collision of quasi-particles. 
The behavior of the large wave may be the easiest to determine an analytical expression for the phase shifts for, as its amplitude is relatively unchanged due to interaction ${ }^{11}$ and none of the spheres around it lose contact. In a similar fashion to the KdV soliton, the two limiting regimes of $V_{l} \gg V_{s}$ and $V_{l} \sim V_{s}$ exhibit very different behavior. For the former, the large wave completely overlaps the small wave (figure $3 \mathrm{a}$ ) while the latter acts more like a collision of two quasiparticles (figure 3b). In contrast, the behavior of the small solitary wave's delay features additional complications due to energy exchange and the formation of secondary solitary waves. As Zhen-Ying, et al. have noted, the amplitude of this wave decays by a few percent due to interaction (its energy goes into forming the secondary solitary waves) and the chain behind the point of interaction fragments, which would lead to a greater delay of the smaller wave than expected as there are no masses to help push the wave forward.

\subsection{Experimental results}

Our experimental results show the exchange of the relative positions of the solitary waves (figure 4) and qualitatively agree with the numerical predictions regarding the phase advances and delays of the solitary waves. The results are presented in figure 5, along with simulated results (without damping). For the large wave, we see that the time advance is close to the predicted numerical results, while for the small the delays are longer than expected (by a few tens of microseconds), likely due to dissipation.

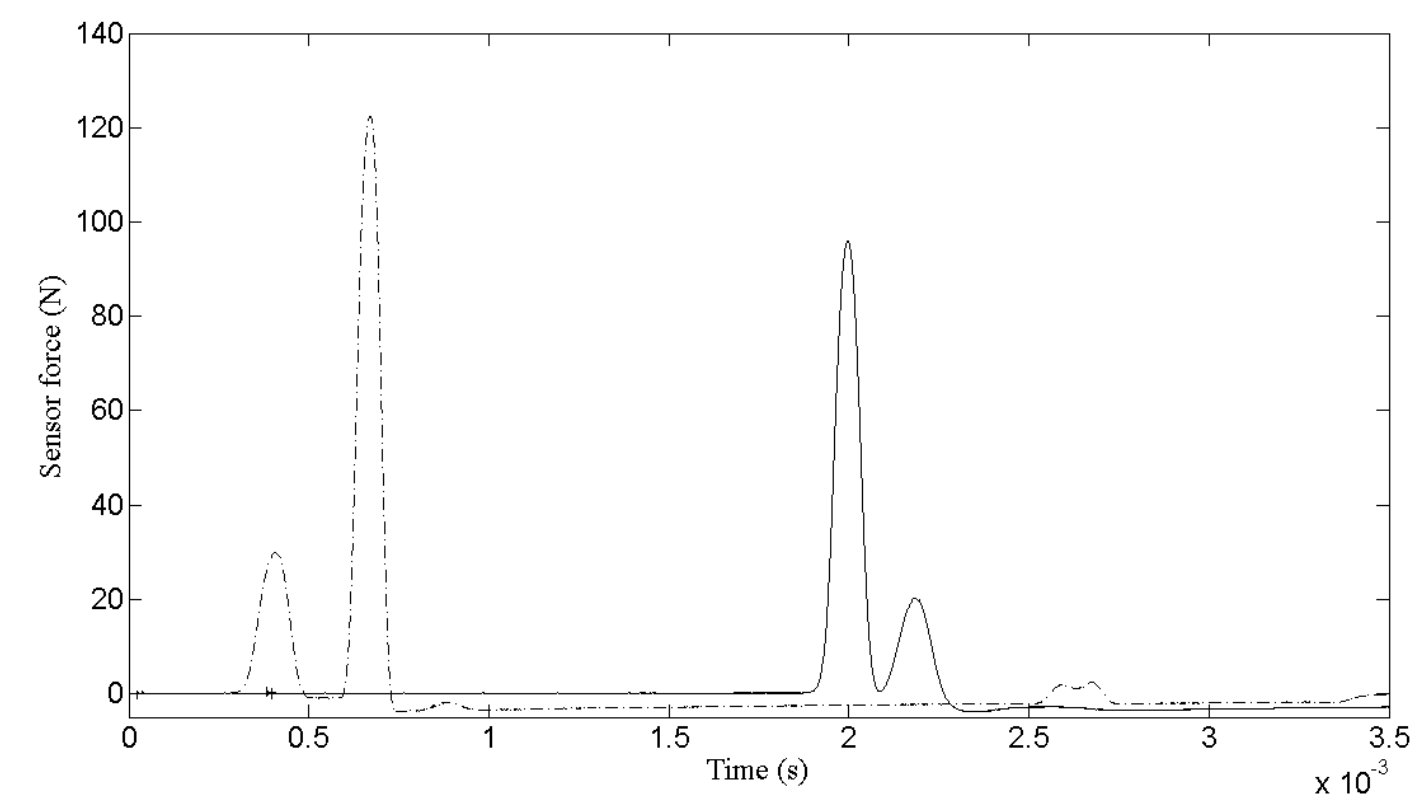

Figure 4: Experimental observation of solitary wave collision. Dashed line is the sensor at 7, solid line is the sensor at 45.

Finally, we observe the presence of secondary solitary waves generated by the wave interaction in almost all of the interactions (see figure 6). These wave amplitudes are close to computational predictions, though a few data sets show secondary waves at significantly larger forces (2-4 N). Santibanez, et al. have suggested that these outliers are due to the presence of frictional forces between the spheres and its supporting structure and small rotations of the spheres. 

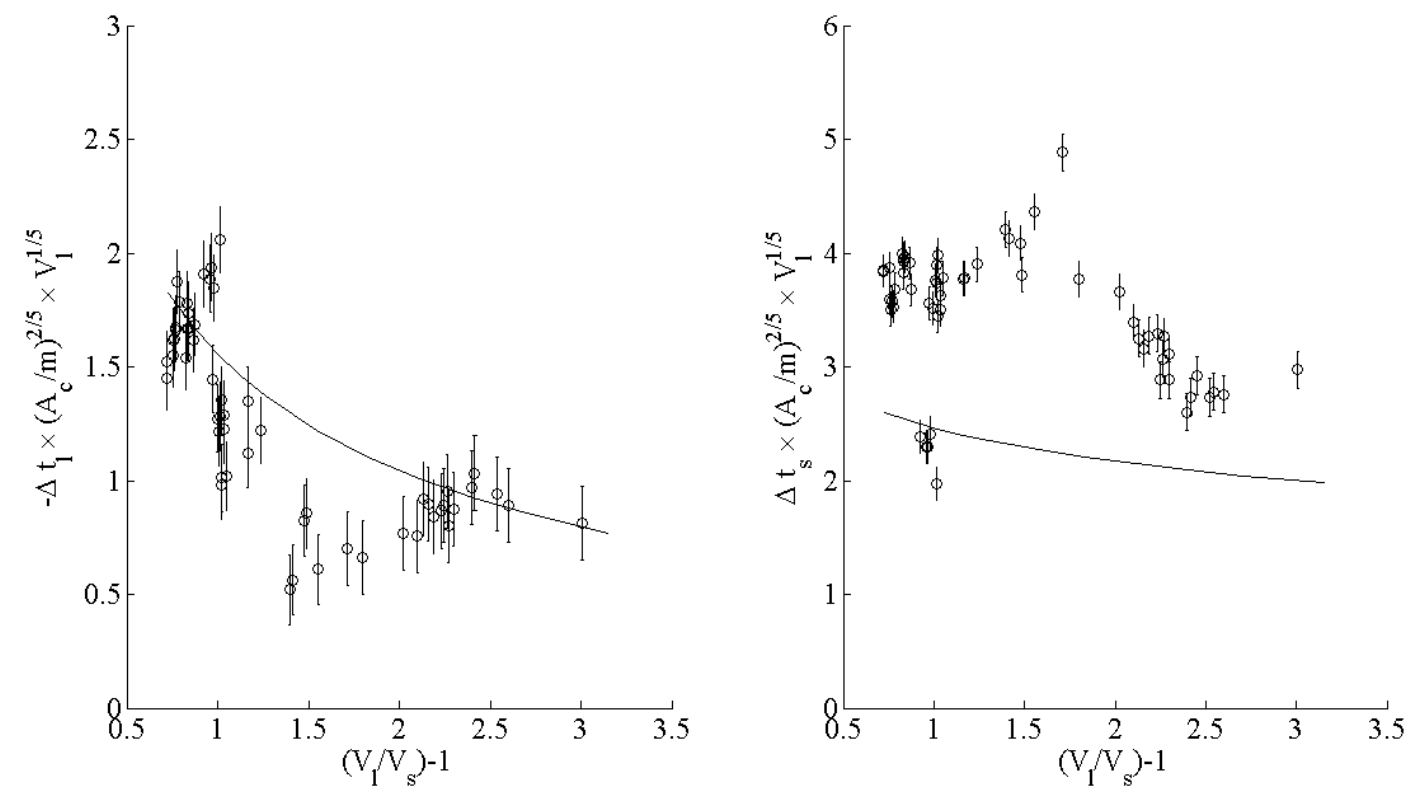

Figure 5: Experimental observation of solitary wave time advances for the large wave (a) and delays for the small wave (b). Circles represent experimental measurements, the line is numerical predictions (without damping).
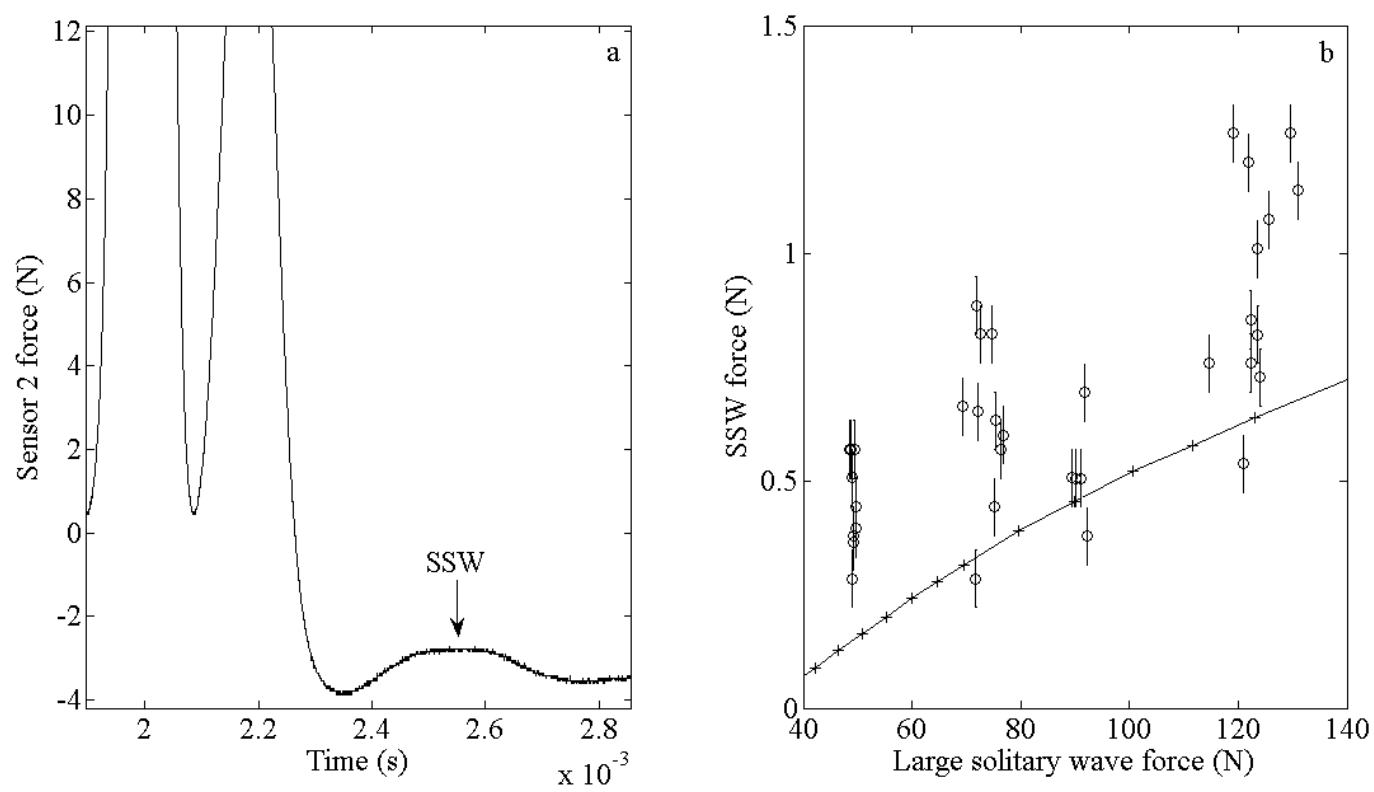

Figure 6: (a) Second solitary wave generated by interaction. This is the same data as seen in figure 4. (b) Observed amplitudes of secondary solitary waves at the second sensor. Dots represent experimental values, the line numerical predictions.

\section{CONCLUSIONS AND DISCUSSION}

In this manuscript, we have investigated the time advance and delay experienced by solitary waves in the Hertzian chain during a co-travelling collision. We have obtained numerical results for the advances of the large wave over 4 decades of 
relative size between the two waves and more than 3 decades for the small wave. We have conducted experiments which confirm that the solitary waves undergo phase advances and delays, as well as the creation of secondary solitary waves due to interaction.

Recent research has begun looking at using granular chains in a variety of NDE and SHM fields, such as analyzing bone stiffness $^{19}$, looking for composite delamination ${ }^{20}$, and monitoring of concrete ${ }^{21}$. These tests are generally actuated by dropping a striker mass on the chain, which requires substantial time to reset between runs and gives the chain plenty of time to completely damp down and resettle. Our method of pulsed piezo-stacks would allow for high-frequency actuation, but solitary-wave interactions could confound results at in this mode without an understanding of their collisions. This work is also of particular interest for the development of an acoustic lens based on the use of granular chains as impulse carriers ${ }^{22}$. The operation of this device requires precisely timing the transmission of solitary waves through several chains, and operation at moderate to high frequencies may similarly lead to solitary wave interactions, which can disrupt the timing if not properly accounted for.

Future work will focus on determining an analytical solution for the time advance of the large solitary wave, and determining whether a similar solution can be found for the small wave's delay. Work to reduce the experimental noise and increase the measured region of $\mathrm{V}_{1} / \mathrm{V}_{\mathrm{s}}$ will be undertaken as well, as will analysis of wave interaction as the spheres are pre-compressed.

\section{ACKNOWLEDGEMENTS}

C.D. acknowledges support for this work from the ONR YIP grant N00014-10-1-0718 and DURIP grant W911NF-10-10201. P.A. is supported by the NASA Space Technology Research Fellowship \#NNX11AN65H. We would like to thank Professor Michael Cross for useful discussions.

\section{REFERENCES}

[1] Nesterenko, V. F., "Propagation of nonlinear compression pulses in granular media," J. Applied Mech. And Theo. Phys. 24(5), 733-743, (1983).

[2] Lazaridi, A. N. and Nesterenko, V. F. "Observation of a new type of solitary waves in a one-dimensional granular medium,” J. Applied Mech. And Theo. Phys. 26(3) 405-408, (1985).

[3] Nesterenko, V. F. [Dynamics of Heterogeneous Materials], Springer, chapter 1, (2001).

[4] Hertz, H. "On the contact of elastic solids", Journal für die Reine und Angewandte Mathematik, 92, 156-171, (1881).

[5] Job, S., et al. "How Hertzian solitary waves interact with boundaries in a 1D granular medium", Phys. Rev. Lett., 94, 178002 (2005).

[6] Ramirez, R., Pöschel, T., Brilliantov, N. V. and Schwager, T. "Coefficient of restitution of colliding viscoelastic spheres" Phys. Rev. E, 60(04), 4465-4472, (1999).

[7] Rosas, A., Romero, Nesterenko, V. F. and Lindenberg K. "Observation of two-wave structure in strongly nonlinear dissipative granular chains”, Phys. Rev. Lett., 98, 164301, (2007).

[8] Carretero-Gonzalez, R., et al., "Dissipative solitary waves in periodic granular media", Phys. Rev. Lett., 102, 024102, (2009).

[9] Manciu, M., Sen, S., and Hurd, A. "Crossing of identical solitary waves in a chain of elastic beads" Phys. Rev. E, 63, 016614, (2000).

[10] Santibanez, F, et al. "Experimental Evidence of Solitary Wave Interactions in Hertzian Chains" Phys. Rev. E 84, 026604 (2011).

[11] Zhen-Ying, W., Shun-Jin, W., Ziu-Ming, Z., and Lei, L. "Solitary Wave Interactions in Granular Media", Chinese Phys. Lett., 24(10), 2887-2890, (2007).

[12] http://www.bearingworks.com/technical_data/ring_and_ball_materials.php,

http://www.azom.com/article.aspx?ArticleID=1024 
[13] Daraio, C., Nesterenko, V.F., Herbold, E., and Jin, S.., "Strongly nonlinear waves in a chain of Teflon beads", Phys. Rev. E, 72, 016603 (2005).

[14] Avalos, E. and Sen, S. "How solitary waves collide in discrete granular alignments" Phys. Rev. E, 79, 046607, (2009).

[15] Nesterenko, V.F., Daraio, C., Herbold, E.B., and Jin, S. "Anomalous wave reflection at the interface of two strongly nonlinear granular media", Phys. Rev. Lett., 95, 158702, (2005).

[16] Preumont, A. [Mechatronics: Dynamics of Electromechanical and Piezoelectric Systems], Springer, New York, Chapter 4, (2006).

[17] Jackson, E. A. [Perspectives of nonlinear dynamics], Vol. 2, Cambridge University Press, Cambridge, Chapter 8, (1990).

[18] Chatterjee, A. "Asymptotic solution for solitary waves in a chain of elastic spheres", Phys. Rev. E, 59(05), 59125919, (1999).

[19] Yang, J, et al., "Nondestructive evaluation of orthopedic implant stability in THA using highly nonlinear solitary waves", Smart Mater. Struct., 21(1), 012002, (2012).

[20] Yang, J., Restuccia F., and Daraio, C., "Highly nonlinear granular crystal sensor and actuator for delamination detection in composite structures", Proc. of Intl. Work. SHM, to appear (2011).

[21] Ni, X., Rizzo, P., and Daraio, C. "Novel Sensor Technology for NDE of Concrete", Proc. SPIE, 7292, 729218, (2009).

[22] Spadoni, A.; Daraio, C. "Generation and control of sound bullets with a nonlinear acoustic lens", PNAS, 107(16), 7230-7234, (2010) 